\title{
Pullback Exponential Attractors for Nonautonomous Klein-Gordon-Schrödinger Equations on Infinite Lattices
}

\author{
Chunqiu Li, ${ }^{1}$ Min Zhao, ${ }^{2}$ and Caidi Zhao ${ }^{1}$ \\ ${ }^{1}$ College of Mathematics and Information Science, Wenzhou University, Wenzhou, Zhejiang 325035, China \\ ${ }^{2}$ College of Life and Environmental Science, Wenzhou University, Wenzhou, Zhejiang 325035, China \\ Correspondence should be addressed to Caidi Zhao; zhaocaidi2013@163.com
}

Received 8 April 2013; Accepted 13 June 2013

Academic Editor: Alberto Parmeggiani

Copyright (c) 2013 Chunqiu Li et al. This is an open access article distributed under the Creative Commons Attribution License, which permits unrestricted use, distribution, and reproduction in any medium, provided the original work is properly cited.

\begin{abstract}
This paper proves the existence of the pullback exponential attractor for the process associated to the nonautonomous Klein-
\end{abstract} Gordon-Schrödinger equations on infinite lattices.

\section{Introduction}

Lattice dynamical systems (LDSs for short), including coupled ordinary differential equations (ODEs), coupled map lattices, and cellular automata [1], are spatiotemporal systems with discretization in some variables. In some cases, LDSs arise as the spatial discretization of partial differential equations (PDEs) on unbounded or bounded domains. LDSs occur in a wide variety of applications, ranging from image processing and pattern recognition [2-4] to electrical engineering [5], chemical reaction theory $[6,7]$, laser systems [8], material science [9], biology [10], and so forth.

Nowadays, LDSs have drawn much attention from mathematicians and physicists [1]. Various properties of solutions for LDSs have been widely studied. For example, the stochastic LDSs were investigated in $[11,12]$. The global and uniform attractors of LDSs were examined in [13-19]. The exponential and uniform exponential attractors of LDSs were investigated by [20-24].

At the same time, the asymptotic theory of LDSs has been widely used on many concrete lattice equations from mathematical physics. For example, lattice reaction-diffusion equations [25], discrete nonlinear Schrödinger equations [26], lattice FitzHugh-Nagumo systems [27], lattice KleinGordon-Schrödinger (KGS) equations [28], and lattice three component reversible Gray-Scott equations [29].

Very recently, Zhou and Han [30] presented some sufficient conditions for the existence of the pullback exponential attractor for the continuous process on Banach spaces and weighted spaces of infinite sequences. Also, they applied their results to study the existence of pullback exponential attractors for first-order nonautonomous differential equations and partly dissipative differential equations on infinite lattices with time-dependent coupled coefficients and timedependent external terms in weighted spaces.

In this paper, we will use the abstract theory of [30] to study the pullback exponential behavior of solutions for the following nonautonomous lattice systems:

$$
\begin{aligned}
& i \dot{z}_{m}-(A z)_{m}+i \alpha z_{m}+z_{m} u_{m}=f_{m}(t), \quad m \in \mathbb{Z}, t>\tau, \\
& \ddot{u}_{m}+v \dot{u}_{m}+(A u)_{m}+\mu u_{m}-\beta\left|z_{m}\right|^{2}=g_{m}(t), \quad m \in \mathbb{Z}, t>\tau,
\end{aligned}
$$

with initial conditions:

$$
\begin{array}{ll}
z_{m}(\tau)=z_{m, \tau}, \quad u_{m}(\tau)=u_{m, \tau}, \quad \dot{u}_{m}(\tau)=u_{1 m, \tau}, \\
\\
m \in \mathbb{Z}, \tau \in \mathbb{R},
\end{array}
$$

where $A$ is a linear operator defined as

$$
(A u)_{m}=2 u_{m}-u_{m+1}-u_{m-1}, \quad \forall u=\left(u_{m}\right)_{m \in \mathbb{Z}},
$$

$z_{m}(t) \in \mathbb{C}, u_{m}(t) \in \mathbb{R}, \mathbb{C}$ and $\mathbb{R}$ are the sets of complex and real numbers, respectively, $\mathbb{Z}$ is the set of integer numbers, $i=\sqrt{-1}$ is the unit of the imaginary numbers, and $\alpha, \beta, v$, and $\mu$ are positive constants. 
Equations (1)-(2) can be regarded as a discrete analogue of the following nonautonomous KGS equations on $\mathbb{R}$ :

$$
\begin{gathered}
i z_{t}+z_{x x}+i \alpha z+z u=f(x, t), \\
u_{t t}+v u_{t}-u_{x x}+\mu u-\beta|z|^{2}=g(x, t) .
\end{gathered}
$$

Equations (5) describe the interaction of a scalar nucleon interacting with a neutral scalar meson through Yukawa coupling [31], where $z$ and $u$ represent the complex scalar nucleon field and the real meson field, respectively, and the complex-valued function $f(x, t)$ and the real-valued function $g(x, t)$ are the time-dependent external sources. There are many works concerning the Cauchy problem and the initial boundary value problem of the continuous model of KGS equations or its related version, see [32-36] and references therein.

We want to mention that the lattice KGS equations have been studied by $[28,37]$. In [37], the authors first presented some sufficient conditions for the existence of the uniform exponential attractor for a family of continuous processes on separable Hilbert spaces and the space of infinite sequences. Then, they studied the existence of uniform exponential attractors for the dissipative nonautonomous KGS lattice system and the Zakharov lattice system driven by quasiperiodic external forces. In [28], the authors first proved the existence of compact kernel sections and gave an upper bound of the Kolmogorov $\varepsilon$-entropy for these kernel sections. Also they verified the upper semicontinuity of the kernel sections. Articles in $[28,37]$ use the same transformation of the variable $u=\left(u_{m}\right)_{m \in \mathbb{Z}}$.

The aim of the present paper is to use the abstract result of [30] to prove the existence of pullback exponential attractors for the LDSs (1)-(3). When verifying the discrete squeezing property (see Lemma 5(II)) of the generated process, we encounter the difficulty coming from the nonlinear terms $u_{m} z_{m}$ and $\left|z_{m}\right|^{2}$ in the coupled lattice equations. To overcome this difficulty, we make a proper transformation of the variable $u=\left(u_{m}\right)_{m \in \mathbb{Z}}$ and use the technique of cutoff functions. We want to remark that the idea concerning the transformation of the variable $u$ originates from articles in $[28,37]$, but our transformation is other than that of $[28,37]$.

The rest of the paper is organized as follows. In Section 2, we first introduce some spaces and operators. Then, we recall some results on the existence, uniqueness, and some estimates of solutions. Section 3 is devoted to proving the existence of the pullback exponential attractor for the process associated to the lattice KGS equations.

\section{Preliminaries}

We first introduce the mathematical setting of our problem. Set

$$
\begin{array}{r}
\ell^{2}=\left\{u=\left(u_{m}\right)_{m \in \mathbb{Z}}, u_{m} \in \mathbb{R}: \sum_{m \in \mathbb{Z}} u_{m}^{2}<+\infty\right\}, \\
L^{2}=\left\{u=\left(u_{m}\right)_{m \in \mathbb{Z}}, u_{m} \in \mathbb{C}: \sum_{m \in \mathbb{Z}}\left|u_{m}\right|^{2}<+\infty\right\} .
\end{array}
$$

For brevity, we use $X$ to denote $\ell^{2}$ or $L^{2}$, and equip $X$ with the inner product and norm as

$$
\begin{gathered}
(u, v)=\sum_{m \in \mathbb{Z}} u_{m} \bar{v}_{m}, \quad\|u\|^{2}=(u, u), \\
\forall u=\left(u_{m}\right)_{m \in \mathbb{Z}}, \quad v=\left(v_{m}\right)_{m \in \mathbb{Z}} \in X,
\end{gathered}
$$

where $\bar{v}_{m}$ denotes the conjugate of $v_{m}$. For any two elements $u, v \in X$, we define a bilinear form on $X$ by

$$
(u, v)_{\mu}=(B u, B v)+\mu(u, v)
$$

where $\mu$ is the constant in (2) and $B$ is a linear operator defined as

$$
(B u)_{m}=u_{m+1}-u_{m}, \quad \forall m \in \mathbb{Z}, \forall u=\left(u_{m}\right)_{m \in \mathbb{Z}} \in X .
$$

We also define a linear operator $B^{*}$ from $X$ to $X$ via

$$
\left(B^{*} u\right)_{m}=u_{m-1}-u_{m}, \quad \forall m \in \mathbb{Z}, \forall u=\left(u_{m}\right)_{m \in \mathbb{Z}} \in X .
$$

In fact, $B^{*}$ is the adjoint operator of $B$ and one can check that

$$
\begin{gathered}
(A u, v)=\left(B^{*} B u, v\right)=(B u, B v), \quad(B u, v)=\left(u, B^{*} v\right), \\
\forall u, v \in X, \\
\|B u\|^{2}=\left\|B^{*} u\right\|^{2} \leq 4\|u\|^{2}, \quad\|A u\|^{2} \leq 16\|u\|^{2}, \\
\forall u \in X .
\end{gathered}
$$

Clearly, the bilinear form $(\cdot, \cdot)_{\mu}$ defined by $(8)$ is also an inner product in $X$. Since

$$
\mu\|u\|^{2} \leq \mu\|u\|^{2}+\|B u\|^{2}=\|u\|_{\mu}^{2} \leq(\mu+4)\|u\|^{2}, \quad \forall u \in X,
$$

the norm $\|\cdot\|_{\mu}$ induced by $(\cdot, \cdot)_{\mu}$ is equivalent to the norm $\|\cdot\|$. Write

$$
\begin{aligned}
& \ell^{2}=\left(\ell^{2},(\cdot, \cdot),\|\cdot\|\right), \\
& \ell_{\mu}^{2}=\left(\ell^{2},(\cdot, \cdot)_{\mu},\|\cdot\|_{\mu}\right), \\
& L^{2}=\left(L^{2},(\cdot, \cdot),\|\cdot\|\right) ;
\end{aligned}
$$

then $\ell^{2}, \ell_{\mu}^{2}$, and $L^{2}$ are all Hilbert spaces. Set

$$
E_{\mu}=\ell_{\mu}^{2} \times \ell^{2} \times L^{2}
$$

For any two elements $\psi^{(j)}=\left(u^{(j)}, v^{(j)}, z^{(j)}\right)^{T} \in E_{\mu}, j=$ 1,2 , the inner product and norm of $E_{\mu}$ are defined as

$$
\begin{gathered}
\left(\psi^{(1)}, \psi^{(2)}\right)_{E_{\mu}=}\left(u^{(1)}, u^{(2)}\right)_{\mu}+\left(v^{(1)}, v^{(2)}\right)+\left(z^{(1)}, z^{(2)}\right) \\
=\sum_{m \in \mathbb{Z}}\left(\left(B u^{(1)}\right)_{m}\left(B u^{(2)}\right)_{m}+\mu u_{m}^{(1)} u_{m}^{(2)}\right. \\
\left.\quad+v_{m}^{(1)} v_{m}^{(2)}+z_{m}^{(1)} \overline{z_{m}^{(2)}}\right), \\
\|\psi\|_{E_{\mu}}^{2}=(\psi, \psi)_{E_{\mu}}, \quad \forall \psi \in E_{\mu},
\end{gathered}
$$


For convenience, we will express (1)-(3) as an abstract Cauchy problem of first-order ODE with respect to time $t$ in $E_{\mu}$. To this end, we put $u=\left(u_{m}\right)_{m \in \mathbb{Z}}, z=\left(z_{m}\right)_{m \in \mathbb{Z}}$, $z u=\left(z_{m} u_{m}\right)_{m \in \mathbb{Z}},|z|^{2}=\left(\left|z_{m}\right|^{2}\right)_{m \in \mathbb{Z}}, f(t)=\left(f_{m}(t)\right)_{m \in \mathbb{Z}}$, $g(t)=\left(g_{m}(t)\right)_{m \in \mathbb{Z}}, z_{\tau}=\left(z_{m, \tau}\right)_{m \in \mathbb{Z}}$, and $u_{\tau}=\left(u_{m, \tau}\right)_{m \in \mathbb{Z}}$, $u_{1 \tau}=\left(u_{1 m, \tau}\right)_{m \in \mathbb{Z}}$. Then, we rewrite (1)-(3) in a vector form as

$$
\begin{gathered}
i \dot{z}-A z+i \alpha z+z u=f(t), \quad t>\tau, \\
\ddot{u}+v \dot{u}+A u+\mu u-\beta|z|^{2}=g(t), \quad t>\tau, \\
z(\tau)=z_{\tau}, \quad u(\tau)=u_{\tau}, \quad \dot{u}(\tau)=u_{1 \tau}, \quad \tau \in \mathbb{R} .
\end{gathered}
$$

Set

$$
\begin{aligned}
& v=\dot{u}+\delta u, \quad \text { where } \delta=\frac{\mu \nu}{2\left(\mu+\nu^{2}\right)}>0, \\
& \psi=(u, v, z)^{T}, F(\psi, t)=\left(0, \beta|z|^{2}+g(t), i z u-i f(t)\right)^{T}, \\
& \Theta=\left(\begin{array}{ccc}
\delta I & -I & 0 \\
A+\mu I+\delta(\delta-\nu) I & (\nu-\delta) I & 0 \\
0 & 0 & i A+\alpha I
\end{array}\right) .
\end{aligned}
$$

Then, (16)-(18) can be written as

$$
\dot{\psi}+\Theta \psi=F(\psi, t), \quad t>\tau,
$$

$$
\psi(\tau)=\psi_{\tau}=\left(u_{\tau}, v_{\tau}, z_{\tau}\right)^{T}=\left(u_{\tau}, u_{1 \tau}+\delta u_{\tau}, z_{\tau}\right)^{T}, \quad \tau \in \mathbb{R} .
$$

Let $\mathscr{C}_{b}(\mathbb{R}, X)$ be the set of continuous and bounded functions from $\mathbb{R}$ into $X$, then for each $f(t) \in \mathscr{C}_{b}(\mathbb{R}, X)$, we have $\sup _{t \in \mathbb{R}} \sum_{m \in \mathbb{Z}}\left|f_{m}(t)\right|^{2}<+\infty$. Write

$$
\begin{aligned}
& \mathscr{H}=\left\{f(t)=\left(f_{m}(t)\right)_{m \in \mathbb{Z}} \in \mathscr{C}_{b}(\mathbb{R}, X):\right. \\
& \text { for each } \tau \in \mathbb{R}, \forall \varepsilon>0, \exists M(\varepsilon, \tau) \in \mathbb{N}, \\
& \text { such that } \sum_{|m| \geq M(\varepsilon, \tau)}\left|f_{m}(s)\right|^{2} \leq \varepsilon \\
& \text { for any } s \leq \tau\} .
\end{aligned}
$$

We next recall some results of solutions to (21)-(22).

Lemma 1 (see [28]). Let $f(t)=\left(f_{m}(t)\right)_{m \in \mathbb{Z}} \in \mathscr{C}_{b}\left(\mathbb{R}, L^{2}\right)$, $g(t)=\left(g_{m}(t)\right)_{m \in \mathbb{Z}} \in \mathscr{C}_{b}\left(\mathbb{R}, \ell^{2}\right)$. Then, for any initial data $\psi_{\tau}=\left(u_{\tau}, v_{\tau}, z_{\tau}\right)^{T} \in E_{\mu}$, there exists a unique solution $\psi(t)=(u(t), v(t), z(t))^{T} \in E_{\mu}$ of (21)-(22), such that $\psi(t) \epsilon$ $\mathscr{C}\left([\tau,+\infty), E_{\mu}\right) \cap \mathscr{C}^{1}\left((\tau,+\infty), E_{\mu}\right)$. Moreover, the mapping

$$
\begin{aligned}
U(t, \tau): \psi_{\tau} & =\left(u_{\tau}, v_{\tau}, z_{\tau}\right)^{T} \in E_{\mu} \longmapsto \psi(t) \\
& =(u(t), v(t), z(t))^{T} \in E_{\mu}, \quad \forall t \geq \tau
\end{aligned}
$$

generates a continuous process $\{U(t, \tau)\}_{t \geq \tau}$ on $E_{\mu}$, where $v_{\tau}=$ $u_{1 \tau}+\delta u_{\tau}$.
Lemma 2 (see [28]). Let $f(t)=\left(f_{m}(t)\right)_{m \in \mathbb{Z}} \in \mathscr{C}_{b}\left(\mathbb{R}, L^{2}\right)$, $g(t)=\left(g_{m}(t)\right)_{m \in \mathbb{Z}} \in \mathscr{C}_{b}\left(\mathbb{R}, \ell^{2}\right)$. Then, the solution $\psi(t)=$ $(u(t), v(t), z(t))^{T} \in E_{\mu}$ of (21)-(22) corresponding to initial data $\psi_{\tau}=\left(u_{\tau}, v_{\tau}, z_{\tau}\right)^{T} \in E_{\mu}$ satisfies

$$
\|\psi(t)\|_{E_{\mu}}^{2} \leq C_{0} \mathrm{e}^{-2 \vartheta_{0}(t-\tau)}+\frac{1}{2} R_{0}^{2}, \quad \forall t \geq \tau,
$$

where $C_{0}, \vartheta_{0}$, and $R_{0}$ are positive constants independent of $t$ and $\tau$.

Lemma 3 (see [28]). Let $f(t)=\left(f_{m}(t)\right)_{m \in \mathbb{Z}} \in \mathscr{C}_{b}\left(\mathbb{R}, L^{2}\right)$, $g(t)=\left(g_{m}(t)\right)_{m \in \mathbb{Z}} \in \mathscr{C}_{b}\left(\mathbb{R}, \ell^{2}\right)$. Then, the process $\{U(t, \tau)\}_{t \geq \tau}$ corresponding to (21)-(22) possesses a uniformly bounded absorbing set $\mathscr{B}_{0} \subset E_{\mu}$, such that for any bounded set $\mathscr{B}$ of $E_{\mu}$, there exists a time $t(\tau, \mathscr{B}) \geq \tau$ yielding

$$
U(t, \tau) \mathscr{B} \subset \mathscr{B}_{0}, \quad \forall t \geq t(\tau, \mathscr{B}),
$$

where $\mathscr{B}_{0}=\mathscr{B}\left(0, R_{0}\right) \subset E_{\mu}$ is a closed ball centered at 0 with radius $R_{0}$.

Lemma 3 shows that there exists a time $t_{0} \doteq t_{0}\left(\tau, \mathscr{B}_{0}\right) \geq \tau$, such that

$$
U(t, \tau) \mathscr{B}_{0} \subset \mathscr{B}_{0}, \quad \forall t \geq t_{0} .
$$

Lemma 4 (see [28]). Let $f(t)=\left(f_{m}(t)\right)_{m \in \mathbb{Z}} \in \mathscr{H}$ with $X=L^{2}$ and $g(t)=\left(g_{m}(t)\right)_{m \in \mathbb{Z}} \in \mathscr{H}$ with $X=\ell^{2}$, respectively. Then, for any $\varepsilon>0$, there exist $t^{*} \doteq t\left(\varepsilon, \mathscr{B}_{0}\right)>t_{0}$ and $M_{0}\left(\varepsilon, \tau, \mathscr{B}_{0}\right) \in$ $\mathbb{N}$, such that when $t \geq t^{*}$, the solution $U(t+\tau, \tau) \psi_{\tau}=\psi(t+\tau)=$ $\left(\psi_{m}(t+\tau)\right)_{m \in \mathbb{Z}} \in E_{\mu}$ of (21)-(22) with $\psi_{\tau} \in \mathscr{B}_{0}$ satisfies

$$
\begin{aligned}
& \sum_{|m|>M_{0}\left(\varepsilon, \tau, \mathscr{B}_{0}\right)}\left|\left(U(t+\tau, \tau) \psi_{\tau}\right)_{m}\right|_{E_{\mu}}^{2} \\
& =\sum_{|m|>M_{0}\left(\varepsilon, \tau, \mathscr{B}_{0}\right)}\left|\psi_{m}(t+\tau)\right|_{E_{\mu}}^{2} \leq \varepsilon^{2},
\end{aligned}
$$

where $\left|\psi_{m}\right|_{E_{\mu}}^{2}=\left|(B u)_{m}\right|^{2}+\mu u_{m}^{2}+v_{m}^{2}+\left|z_{m}\right|^{2}$.

\section{Existence of the Pullback Exponential Attractors}

In this section, we prove the existence of the pullback exponential attractor for the process $\{U(t, \tau)\}_{t \geq \tau}$ defined by (24). Write

$$
E_{\mu}^{(N)}=\left\{\psi=\left(\psi_{m}\right)_{m \in \mathbb{Z}} \in E_{\mu} \mid \psi_{m}=(0,0,0)^{T} \text { if }|m|>N\right\},
$$

then $E_{\mu}^{(N)}$ is a $4(2 N+1)$-dimensional subspace of $E_{\mu}$. Define a bounded projection $P_{N}: E_{\mu} \mapsto E_{\mu}^{(N)} \subset E_{\mu}$ by

$$
\left(P_{N} \psi\right)_{m}=\left\{\begin{array}{ll}
\psi_{m}, & |m| \leq N ; \\
0, & |m|>N,
\end{array} \quad \psi=\left(\psi_{m}\right)_{m \in \mathbb{Z}} \in E_{\mu} .\right.
$$


Lemma 5. (I) For any $T>t_{0}$, there exists some $L_{T}>$ 0 (independent of $\tau$ ), such that for every $\tau \in \mathbb{R}$ and any $t \in\left[t_{0}, T\right]$,

$$
\begin{aligned}
\| U(t & +\tau, \tau) \psi_{\tau}^{(1)}-U(t+\tau, \tau) \psi_{\tau}^{(2)} \|_{E_{\mu}} \\
& \leq L_{T}\left\|\psi_{\tau}^{(1)}-\psi_{\tau}^{(2)}\right\|_{E_{\mu}}, \quad \forall \psi_{\tau}^{(1)}, \psi_{\tau}^{(2)} \in \mathscr{B}_{0} .
\end{aligned}
$$

(II) There exist two positive constants $T^{*}>t_{0}$ and $\gamma \in$ $(0,1 / 2)$, and a $4\left(2 N^{*}+1\right)$-dimensional orthogonal projection $P_{N^{*}}: E_{\mu} \mapsto E_{\mu}^{\left(N^{*}\right)}$ for some $N^{*} \in \mathbb{N}$, such that for every $\tau \in \mathbb{R}$ and $\psi_{\tau}^{(1)}, \psi_{\tau}^{(2)} \in \mathscr{B}_{0}$,

$$
\begin{aligned}
& \left\|\left(I-P_{N^{*}}\right)\left[U\left(T^{*}+\tau, \tau\right) \psi_{\tau}^{(1)}-U\left(T^{*}+\tau, \tau\right) \psi_{\tau}^{(2)}\right]\right\|_{E_{\mu}} \\
& \quad \leq \gamma\left\|\psi_{\tau}^{(1)}-\psi_{\tau}^{(2)}\right\|_{E_{\mu}} .
\end{aligned}
$$

Proof. (I) For any $\tau \in \mathbb{R}$, let

$$
\begin{gathered}
\psi^{(1)}(t)=U(t, \tau) \psi_{\tau}^{(1)}, \\
\psi^{(2)}(t)=U(t, \tau) \psi_{\tau}^{(2)}, \quad \forall t \geq \tau
\end{gathered}
$$

be two solutions of (21)-(22) with initial conditions $\psi_{\tau}^{(1)}$, $\psi_{\tau}^{(2)} \in \mathscr{B}_{0}$, respectively. Set

$$
\begin{array}{ll}
u_{d}(t)=u^{(1)}(t)-u^{(2)}(t), & v_{d}(t)=v^{(1)}(t)-v^{(2)}(t), \\
z_{d}(t)=z^{(1)}(t)-z^{(2)}(t), & \psi_{d}(t)=\psi^{(1)}(t)-\psi^{(2)}(t) .
\end{array}
$$

From (21)-(22), we get

$$
\begin{gathered}
\dot{\psi}_{d}+\Theta \psi_{d}=F\left(\psi^{(1)}, t\right)-F\left(\psi^{(2)}, t\right), \quad \forall t>\tau . \\
\psi_{d}(\tau)=\psi_{\tau}^{(1)}-\psi_{\tau}^{(2)} .
\end{gathered}
$$

Taking the real part of the inner product of (35) with $\psi_{d}$ in $E_{\mu}$, we obtain

$$
\begin{aligned}
\frac{1}{2} \frac{\mathrm{d}}{\mathrm{d} t}\left\|\psi_{d}\right\|_{E_{\mu}}^{2}+\operatorname{Re}\left(\Theta \psi_{d}\right. & +F\left(\psi^{(2)}, t\right) \\
- & \left.F\left(\psi^{(1)}, t\right), \psi_{d}\right)_{E_{\mu}}=0, \quad \forall t \geq \tau .
\end{aligned}
$$

Since $\Theta: E_{\mu} \mapsto E_{\mu}$ is a bounded linear operator, $F$ : $E_{\mu} \times \mathbb{R} \mapsto E_{\mu}$ is a locally Lipschitz continuous operator (see Lemma 2.2 in [28]), and $\mathscr{B}_{0}$ is a bounded set in $E_{\mu}$, we see that there exist two positive constants $C_{1}$ and $K_{1}=K_{1}\left(\mathscr{B}_{0}\right)$, such that

$$
\begin{aligned}
& \operatorname{Re}\left(\Theta \psi_{d}+F\left(\psi^{(2)}, t\right)-F\left(\psi^{(1)}, t\right), \psi_{d}\right)_{E_{\mu}} \\
& \quad \leq\left(C_{1}\left\|\psi_{d}\right\|_{E_{\mu}}+\left\|F\left(\psi^{(2)}, t\right)-F\left(\psi^{(1)}, t\right)\right\|_{E_{\mu}}\right)\left\|\psi_{d}\right\|_{E_{\mu}} \\
& \leq\left(C_{1}+K_{1}\right)\left\|\psi_{d}\right\|_{E_{\mu}}^{2}, \quad \forall t \geq \tau .
\end{aligned}
$$

Combining (36) and (37), we get

$$
\frac{\mathrm{d}}{\mathrm{d} t}\left\|\psi_{d}\right\|_{E_{\mu}}^{2}-K_{2}\left\|\psi_{d}\right\|_{E_{\mu}}^{2} \leq 0, \quad \forall t \geq \tau
$$

where $K_{2}=2\left(C_{1}+K_{1}\right)$. Applying Gronwall inequality to (38) on $[\tau, \tau+t]$ with $t \in\left[t_{0}, T\right]$, we obtain

$$
\left\|\psi_{d}(t+\tau)\right\|_{E_{\mu}}^{2} \leq \mathrm{e}^{K_{2} t}\left\|\psi_{d}(\tau)\right\|_{E_{\mu}}^{2}, \quad \forall t \in\left[t_{0}, T\right] .
$$

Thus,

$$
\begin{aligned}
\left\|\psi_{d}(t+\tau)\right\|_{E_{\mu}} \\
=\left\|\psi^{(1)}(t+\tau)-\psi^{(2)}(t+\tau)\right\|_{E_{\mu}} \\
=\left\|U(t+\tau, \tau) \psi_{\tau}^{(1)}-U(t+\tau, \tau) \psi_{\tau}^{(2)}\right\|_{E_{\mu}} \\
\leq L_{T}\left\|\psi_{\tau}^{(1)}-\psi_{\tau}^{(2)}\right\|_{E_{\mu}}, \quad \forall t \in\left[t_{0}, T\right],
\end{aligned}
$$

where $L_{T}=\sqrt{\mathrm{e}^{K_{2} T}}$ does not depend on $\tau$.

(II) Define a smooth function $\chi(x) \in \mathscr{C}^{1}\left(\mathbb{R}_{+},[0,1]\right)$, such that

$$
\chi(x)= \begin{cases}0, & 0 \leq x \leq 1 \\ 1, & x \geq 2\end{cases}
$$

$\left|\chi^{\prime}(x)\right| \leq \chi_{0}$ (positive constant), $\forall x \in \mathbb{R}_{+}$.

Set

$$
\begin{gathered}
p_{d}=\left(p_{d m}\right)_{m \in \mathbb{Z}} \text { with } p_{d m}=\chi\left(\frac{|m|}{M}\right) u_{d m}, \\
q_{d}=\left(q_{d m}\right)_{m \in \mathbb{Z}} \text { with } q_{d m}=\chi\left(\frac{|m|}{M}\right) v_{d m}, \\
w_{d}=\left(w_{d m}\right)_{m \in \mathbb{Z}} \text { with } w_{d m}=\chi\left(\frac{|m|}{M}\right) z_{d m}, \\
\phi_{d}=\left(\phi_{d m}\right)_{m \in \mathbb{Z}} \text { with } \phi_{d m}=\left(p_{d m}, q_{d m}, w_{d m}\right),
\end{gathered}
$$

where $M$ is a positive integer that will be specified later. From (16), we see that

$$
i \dot{z}_{d}-A z_{d}+i \alpha z_{d}+z^{(1)} u^{(1)}-z^{(2)} u^{(2)}=0
$$

Taking the imaginary part of the inner product of (43) with $w_{d}$ in $L^{2}$, we get

$$
\begin{aligned}
& \frac{1}{2} \frac{\mathrm{d}}{\mathrm{d} t} \sum_{m \in \mathbb{Z}} \chi\left(\frac{|m|}{M}\right)\left|z_{d m}\right|^{2}-\operatorname{Im}\left(A z_{d}, w_{d}\right)+\alpha \sum_{m \in \mathbb{Z}} \chi\left(\frac{|m|}{M}\right)\left|z_{d m}\right|^{2} \\
& +\operatorname{Im}\left(z^{(1)} u^{(1)}-z^{(2)} u^{(2)}, w_{d}\right)=0 .
\end{aligned}
$$


Now, we have

$$
\begin{aligned}
-\operatorname{Im} & \left(A z_{d}, w_{d}\right) \\
& =-\operatorname{Im}\left(B z_{d}, B w_{d}\right) \\
& =-\operatorname{Im}\left(\sum_{m \in \mathbb{Z}}\left(z_{d m+1}-z_{d m}\right)\right. \\
& \times \operatorname{Im}\left(\sum_{m \in \mathbb{Z}}\left(\chi\left(\frac{|m+1|}{M}\right) \bar{z}_{d m+1}-\chi\left(\frac{|m|}{M}\right) \bar{z}_{d m}\right)\right) \\
& =\operatorname{Im}\left(\sum_{m \in \mathbb{Z}}\left(\chi\left(\frac{|m+1|}{M}\right)-\chi\left(\frac{|m|}{M}\right)\right) \bar{z}_{d m+1} z_{d m}\right) \\
\geq & -\sum_{m \in \mathbb{Z}}\left|\chi \chi^{\prime}\left(\frac{\tilde{m}}{M}\right)\right| \frac{1}{M}\left|z_{d m+1}\right|\left|z_{d m}\right| \\
& \left.\left.\left.+\frac{|m+1|}{M}\right) \bar{z}_{d m+1} z_{d m}\right)\right)
\end{aligned}
$$

where $\widetilde{m}$ locates between $|m+1|$ and $|m|$. According to Lemma 4, we know that there exist $t_{1}\left(t_{1}>t_{0}\right)$ and $M_{1}\left(\varepsilon, \tau, \mathscr{B}_{0}\right) \in \mathbb{N}$, such that when $t \geq t_{1}$ and $M>$ $M_{1}\left(\varepsilon, \tau, \mathscr{B}_{0}\right)$, we obtain

$$
\begin{aligned}
\operatorname{Im} \sum_{m \in \mathbb{Z}} & \left(z_{m}^{(1)} u_{m}^{(1)}-z_{m}^{(2)} u_{m}^{(2)}, \chi\left(\frac{|m|}{M}\right)\left(z_{m}^{(1)}-z_{m}^{(2)}\right)\right) \\
\leq & \sum_{m \in \mathbb{Z}} \chi\left(\frac{|m|}{M}\right)\left|z_{m}^{(1)} u_{m}^{(1)}-z_{m}^{(2)} u_{m}^{(2)}\right|\left|z_{m}^{(1)}-z_{m}^{(2)}\right| \\
= & \sum_{m \in \mathbb{Z}} \chi\left(\frac{|m|}{M}\right)\left|z_{m}^{(1)}\left(u_{m}^{(1)}-u_{m}^{(2)}\right)+u_{m}^{(2)}\left(z_{m}^{(1)}-z_{m}^{(2)}\right)\right| \\
& \times\left|z_{m}^{(1)}-z_{m}^{(2)}\right| \\
\leq & \frac{\alpha}{4} \sum_{m \in \mathbb{Z}} \chi\left(\frac{|m|}{M}\right)\left|z_{m}^{(1)}-z_{m}^{(2)}\right|^{2}+\frac{\sqrt{\alpha \mu \delta}}{2} \\
& \times \sum_{m \in \mathbb{Z}} \chi\left(\frac{|m|}{M}\right)\left|u_{m}^{(1)}-u_{m}^{(2)}\right|\left|z_{m}^{(1)}-z_{m}^{(2)}\right| \\
\leq & \frac{\alpha}{2} \sum_{m \in \mathbb{Z}} \chi\left(\frac{|m|}{M}\right)\left|z_{m}^{(1)}-z_{m}^{(2)}\right|^{2} \\
& +\frac{\mu \delta}{4} \sum_{m \in \mathbb{Z}} \chi\left(\frac{|m|}{M}\right)\left|u_{m}^{(1)}-u_{m}^{(2)}\right|^{2},
\end{aligned}
$$

which implies that when $t \geq t_{1}$ and $M>M_{1}\left(\varepsilon, \tau, \mathscr{B}_{0}\right)$

$$
\begin{aligned}
& \operatorname{Im}\left(z^{(1)} u^{(1)}-z^{(2)} u^{(2)}, w_{d}\right) \\
&=\operatorname{Im} \sum_{m \in \mathbb{Z}} \chi\left(\frac{|m|}{M}\right)\left(z_{m}^{(1)} u_{m}^{(1)}-z_{m}^{(2)} u_{m}^{(2)},\left(z_{m}^{(1)}-z_{m}^{(2)}\right)\right) \\
& \geq-\frac{\alpha}{2} \sum_{m \in \mathbb{Z}} \chi\left(\frac{|m|}{M}\right)\left|z_{m}^{(1)}-z_{m}^{(2)}\right|^{2} \\
&-\frac{\mu \delta}{4} \sum_{m \in \mathbb{Z}} \chi\left(\frac{|m|}{M}\right)\left|u_{m}^{(1)}-u_{m}^{(2)}\right|^{2} .
\end{aligned}
$$

Then, taking (44)-(47) into account, we obtain for every $t \geq t_{1}$ and $M>M_{1}\left(\varepsilon, \tau, \mathscr{B}_{0}\right)$ that

$$
\begin{gathered}
\frac{\mathrm{d}}{\mathrm{d} t} \sum_{m \in \mathbb{Z}} \chi\left(\frac{|m|}{M}\right)\left|z_{d m}\right|^{2}+\alpha \sum_{m \in \mathbb{Z}} \chi\left(\frac{|m|}{M}\right)\left|z_{d m}\right|^{2} \\
\leq \frac{\mu \delta}{2} \sum_{m \in \mathbb{Z}} \chi\left(\frac{|m|}{M}\right) u_{d m}^{2}+\frac{4 \chi_{0}}{M}\left\|\psi_{d}\right\|_{E_{\mu}}^{2} .
\end{gathered}
$$

From (17) and (19), we obtain

$$
\begin{aligned}
\dot{v}_{d}+ & (\nu-\delta) v_{d}+(\delta(\delta-\nu)+\mu) u_{d} \\
& +A u_{d}-\beta\left(\left|z^{(1)}\right|^{2}-\left|z^{(2)}\right|^{2}\right)=0 .
\end{aligned}
$$

Taking the inner product of (49) with $q_{d}$ in $\ell^{2}$, we obtain

$$
\begin{aligned}
& \frac{1}{2} \frac{\mathrm{d}}{\mathrm{d} t} \sum_{m \in \mathbb{Z}} \chi\left(\frac{|m|}{M}\right) v_{d m}^{2}+(\nu-\delta) \\
& \quad \times \sum_{m \in \mathbb{Z}} \chi\left(\frac{|m|}{M}\right) v_{d m}^{2}+\left((\delta(\delta-\nu)+\mu) u_{d}, q_{d}\right) \\
& +\left(A u_{d}, q_{d}\right)-\beta\left(\left|z^{(1)}\right|^{2}-\left|z^{(2)}\right|^{2}, q_{d}\right)=0 .
\end{aligned}
$$

$$
\begin{aligned}
& \frac{\mathrm{d}}{\mathrm{d} t} \sum_{m \in \mathbb{Z}} \chi\left(\frac{|m|}{M}\right)\left(\mu u_{d m}^{2}+v_{d m}^{2}\right) \\
& +2(\nu-\delta) \sum_{m \in \mathbb{Z}} \chi\left(\frac{|m|}{M}\right) v_{d m}^{2} \\
& +2 \mu \delta \sum_{m \in \mathbb{Z}} \chi\left(\frac{|m|}{M}\right) u_{d m}^{2} \\
& +2 \delta(\delta-\nu)\left(u_{d}, q_{d}\right)+2\left(A u_{d}, q_{d}\right) \\
& \quad-2 \beta\left(\left|z^{(1)}\right|^{2}-\left|z^{(2)}\right|^{2}, q_{d}\right)=0 .
\end{aligned}
$$

Also, we have

$$
\begin{aligned}
\left(A u_{d}, q_{d}\right) & =\left(A u_{d}, \dot{p}_{d}\right)+\delta\left(A u_{d}, p_{d}\right) \\
& =\left(B u_{d}, B \dot{p}_{d}\right)+\delta\left(B u_{d}, B p_{d}\right) .
\end{aligned}
$$


By some computations, we get

$$
\begin{aligned}
\left(B u_{d},\right. & \left.B \dot{p}_{d}\right) \\
= & \sum_{m \in \mathbb{Z}}\left(B u_{d}\right)_{m}\left(B \dot{p}_{d}\right)_{m} \\
= & \sum_{m \in \mathbb{Z}}\left(B u_{d}\right)_{m}\left[\chi\left(\frac{|m+1|}{M}\right) \dot{u}_{d m+1}-\chi\left(\frac{|m|}{M}\right) \dot{u}_{d m}\right] \\
= & \sum_{m \in \mathbb{Z}}\left(B u_{d}\right)_{m}\left[\left(B \dot{u}_{d}\right)_{m} \chi\left(\frac{|m|}{M}\right)\right. \\
= & \sum_{m \in \mathbb{Z}} \chi\left(\frac{|m|}{M}\right)\left(B u_{d}\right)_{m}\left(B \dot{u}_{d}\right)_{m} \\
& +\frac{2 \chi_{0}(\mu+\delta+1)}{M} \frac{|m+1|}{\mathrm{d} t} \sum_{m \in \mathbb{Z}} \chi\left(\frac{|m|}{M}\right)\left|\left(B u_{d}\right)_{m}\right|_{E_{\mu}}^{2} \quad \forall t \geq \tau \\
& \left.\left.\left.+\frac{|m|}{M}\right)\right) \dot{u}_{d m+1}\right] \\
& \times\left(\frac{\tilde{m}}{M}\right) \frac{1}{M}\left(v_{d m+1}-\delta u_{d m+1}\right) \\
& \left.1 u_{d m}\right)
\end{aligned}
$$

$$
\begin{aligned}
\delta\left(B u_{d}, B p_{d}\right) & \\
= & \delta \sum_{m \in \mathbb{Z}}\left(B u_{d}\right)_{m}\left(B p_{d}\right)_{m} \\
= & \delta \sum_{m \in \mathbb{Z}}\left(B u_{d}\right)_{m}\left[\chi\left(\frac{|m+1|}{M}\right) u_{d m+1}-\chi\left(\frac{|m|}{M}\right) u_{d m}\right] \\
= & \delta \sum_{m \in \mathbb{Z}}\left(B u_{d}\right)_{m}\left[\left(B u_{d}\right)_{m} \chi\left(\frac{|m|}{M}\right)\right. \\
= & \left.\left.\delta \sum_{m \in \mathbb{Z}} \chi\left(\frac{|m|}{M}\right)\left|\left(B u_{d}\right)_{m}\right|^{2}\left(\frac{|m+1|}{M}\right)-\chi\left(\frac{|m|}{M}\right)\right) u_{d m+1}\right] \\
& +\delta \sum_{m \in \mathbb{Z}} \chi^{\prime}\left(\frac{\widetilde{m}}{M}\right) \frac{1}{M}\left(u_{d m+1}-u_{d m}\right) u_{d m+1} \\
\geq & \delta \sum_{m \in \mathbb{Z}} \chi\left(\frac{|m|}{M}\right)\left|\left(B u_{d}\right)_{m}\right|^{2}-\frac{2 \chi_{0} \delta}{M \mu}\left\|\psi_{d}\right\|_{E_{\mu}}^{2}, \forall t \geq \tau .
\end{aligned}
$$

Here $\widetilde{m}$ locates between $|m+1|$ and $|m|$. Inserting (53) into (52), we get

$$
\begin{aligned}
\left(A u_{d}, q_{d}\right) \geq & \frac{1}{2} \frac{\mathrm{d}}{\mathrm{d} t} \sum_{m \in \mathbb{Z}} \chi\left(\frac{|m|}{M}\right)\left|\left(B u_{d}\right)_{m}\right|^{2} \\
& +\delta \sum_{m \in \mathbb{Z}} \chi\left(\frac{|m|}{M}\right)\left|\left(B u_{d}\right)_{m}\right|^{2} \\
& -\frac{2 \chi_{0}(\mu+2 \delta+1)}{M \mu}\left\|\psi_{d}\right\|_{E_{\mu}}^{2}, \quad \forall t \geq \tau .
\end{aligned}
$$

According to Lemma 4, we can see that there exist $t_{2}>t_{1}$ and $M_{2}\left(\varepsilon, \tau, \mathscr{B}_{0}\right)>M_{1}\left(\varepsilon, \tau, \mathscr{B}_{0}\right)$, such that when $t \geq t_{2}$ and $M>M_{2}\left(\varepsilon, \tau, \mathscr{B}_{0}\right)$,

$$
\begin{aligned}
& \beta\left(\left|z^{(1)}\right|^{2}-\left|z^{(2)}\right|^{2}, q_{d}\right) \\
& \quad=\beta \sum_{m \in \mathbb{Z}} \chi\left(\frac{|m|}{M}\right)\left(\left|z_{m}^{(1)}\right|^{2}-\left|z_{m}^{(2)}\right|^{2}\right) v_{d m} \\
& \leq \beta \sum_{m \in \mathbb{Z}} \chi\left(\frac{|m|}{M}\right)\left(\left|z_{m}^{(1)}\right|+\left|z_{m}^{(2)}\right|\right)\left|z_{d m}\right|\left|v_{d m}\right| \\
& \leq \beta \frac{\sqrt{2 \alpha \nu}}{2 \beta} \sum_{m \in \mathbb{Z}} \chi\left(\frac{|m|}{M}\right)\left|z_{d m}\right|\left|v_{d m}\right| \\
& \leq \frac{\nu}{2} \sum_{m \in \mathbb{Z}} \chi\left(\frac{|m|}{M}\right) v_{d m}^{2}+\frac{\alpha}{4} \sum_{m \in \mathbb{Z}} \chi\left(\frac{|m|}{M}\right)\left|z_{d m}\right|^{2} .
\end{aligned}
$$

It follows from (51) and (54)-(55) that when $M>$ $M_{2}\left(\varepsilon, \tau, \mathscr{B}_{0}\right)$ and $t \geq t_{2}$, we have

$$
\begin{aligned}
\frac{\mathrm{d}}{\mathrm{d} t} \sum_{m \in \mathbb{Z}} \chi & \left(\frac{|m|}{M}\right)\left[\left|\left(B u_{d}\right)_{m}\right|^{2}+\mu u_{d m}^{2}+v_{d m}^{2}\right] \\
& +2 \delta \sum_{m \in \mathbb{Z}} \chi\left(\frac{|m|}{M}\right)\left|\left(B u_{d}\right)_{m}\right|^{2} \\
& +2 \mu \delta \sum_{m \in \mathbb{Z}} \chi\left(\frac{|m|}{M}\right) u_{d m}^{2}+(\nu-2 \delta) \\
& \times \sum_{m \in \mathbb{Z}} \chi\left(\frac{|m|}{M}\right) v_{d m}^{2}-2 \delta(\nu-\delta)\left(u_{d}, q_{d}\right) \\
\leq & \frac{\alpha}{2} \sum_{m \in \mathbb{Z}} \chi\left(\frac{|m|}{M}\right)\left|z_{d m}\right|^{2}+\frac{4 \chi_{0}(\mu+2 \delta+1)}{M \mu}\left\|\psi_{d}\right\|_{E_{\mu}}^{2} .
\end{aligned}
$$

Combining (48) and (56), when $t \geq t_{2}$, we obtain

$$
\begin{aligned}
& \frac{\mathrm{d}}{\mathrm{d} t} \sum_{m \in \mathbb{Z}} \chi\left(\frac{|m|}{M}\right)\left[\left|\left(B u_{d}\right)_{m}\right|^{2}+\mu u_{d m}^{2}+v_{d m}^{2}+\left|z_{d m}\right|^{2}\right] \\
& +2 \delta \sum_{m \in \mathbb{Z}} \chi\left(\frac{|m|}{M}\right)\left|\left(B u_{d}\right)_{m}\right|^{2}
\end{aligned}
$$




$$
\begin{aligned}
& +\frac{3 \mu \delta}{2} \sum_{m \in \mathbb{Z}} \chi\left(\frac{|m|}{M}\right) u_{d m}^{2}+(v-2 \delta) \\
& \times \sum_{m \in \mathbb{Z}} \chi\left(\frac{|m|}{M}\right) v_{d m}^{2}+\frac{\alpha}{2} \sum_{m \in \mathbb{Z}} \chi\left(\frac{|m|}{M}\right)\left|z_{d m}\right|^{2} \\
& -2 \delta(\nu-\delta)\left(u_{d}, q_{d}\right) \leq \frac{4 \chi_{0}(2 \mu+2 \delta+1)}{M \mu} \\
& \times\left\|\psi_{d}\right\|_{E_{\mu}}^{2}, \quad \forall M>M_{2}\left(\varepsilon, \tau, \mathscr{B}_{0}\right) .
\end{aligned}
$$

Since $\delta^{2} \nu^{2}=\mu \delta(\nu / 2-\delta)$, we get for any $m \in \mathbb{Z}$ that

$$
\begin{aligned}
\mu \delta u_{d m}^{2} & +\left(\frac{\nu}{2}-\delta\right) v_{d m}^{2}-2 \delta(\nu-\delta) u_{d m} v_{d m} \\
& \geq \mu \delta u_{d m}^{2}+\left(\frac{v}{2}-\delta\right) v_{d m}^{2}-2 \delta \nu\left|u_{d m} v_{d m}\right| \geq 0 .
\end{aligned}
$$

Thus,

$$
\begin{gathered}
\mu \delta \sum_{m \in \mathbb{Z}} \chi\left(\frac{|m|}{M}\right) u_{d m}^{2}+\left(\frac{\nu}{2}-\delta\right) \sum_{m \in \mathbb{Z}} \chi\left(\frac{|m|}{M}\right) v_{d m}^{2} \\
-2 \delta(\nu-\delta)\left(u_{d}, q_{d}\right) \geq 0 .
\end{gathered}
$$

We then conclude from (57) and (59) that when $M>$ $M_{2}\left(\varepsilon, \tau, \mathscr{B}_{0}\right)$ and $t \geq t_{2}$,

$$
\begin{aligned}
\frac{\mathrm{d}}{\mathrm{d} t} \sum_{m \in \mathbb{Z}} \chi & \left(\frac{|m|}{M}\right)\left[\left|\left(B u_{d}\right)_{m}\right|^{2}+\mu u_{d m}^{2}+v_{d m}^{2}+\left|z_{d m}\right|^{2}\right] \\
& +2 \delta \sum_{m \in \mathbb{Z}} \chi\left(\frac{|m|}{M}\right)\left|\left(B u_{d}\right)_{m}\right|^{2} \\
& +\frac{\mu \delta}{2} \sum_{m \in \mathbb{Z}} \chi\left(\frac{|m|}{M}\right) u_{d m}^{2}+\left(\frac{\nu}{2}-\delta\right) \sum_{m \in \mathbb{Z}} \chi\left(\frac{|m|}{M}\right) v_{d m}^{2} \\
& +\frac{\alpha}{2} \sum_{m \in \mathbb{Z}} \chi\left(\frac{|m|}{M}\right)\left|z_{d m}\right|^{2} \\
\leq & \frac{4 \chi_{0}(2 \mu+2 \delta+1)}{M \mu}\left\|\psi_{d}\right\|_{E_{\mu}}^{2} .
\end{aligned}
$$

Choosing $\vartheta=\min \{\delta / 2, \nu / 2-\delta, \alpha / 2\}$, we obtain

$$
\begin{gathered}
\frac{\mathrm{d}}{\mathrm{d} t} \sum_{m \in \mathbb{Z}} \chi\left(\frac{|m|}{M}\right)\left|\psi_{d m}\right|_{E_{\mu}}^{2}+\vartheta \sum_{m \in \mathbb{Z}} \chi\left(\frac{|m|}{M}\right)\left|\psi_{d m}\right|_{E_{\mu}}^{2} \\
\leq \frac{4 \chi_{0}(2 \mu+2 \delta+1)}{M \mu}\left\|\psi_{d}\right\|_{E_{\mu}}^{2}, \\
\forall t \geq t_{2}, \quad \forall M>M_{2}\left(\varepsilon, \tau, \mathscr{B}_{0}\right) .
\end{gathered}
$$

Applying Gronwall inequality to (61) from $\tau+t_{2}$ to $\tau+$ $t$ with $t>t_{2}$, we get for every $\psi_{\tau}^{(1)}, \psi_{\tau}^{(2)} \in \mathscr{B}_{0}, M>$ $M_{2}\left(\varepsilon, \tau, \mathscr{B}_{0}\right)$ and $t \geq t_{2}$ that

$$
\begin{aligned}
\sum_{m \in \mathbb{Z}} \chi\left(\frac{|m|}{M}\right)\left|\psi_{d m}(t+\tau)\right|_{E_{\mu}}^{2} & \leq \mathrm{e}^{-9\left(t-t_{2}\right)} \sum_{m \in \mathbb{Z}} \chi\left(\frac{|m|}{M}\right)\left|\psi_{d m}\left(\tau+t_{2}\right)\right|_{E_{\mu}}^{2} \\
& +\frac{4 \chi_{0}(2 \mu+2 \delta+1)}{M \mu} \int_{\tau+t_{2}}^{\tau+t}\left\|\psi_{d}(s)\right\|_{E_{\mu}}^{2} \mathrm{e}^{-\vartheta(t+\tau-s)} \mathrm{d} s .
\end{aligned}
$$

By (39), when $M>M_{2}\left(\varepsilon, \tau, \mathscr{B}_{0}\right)$ and $t \geq t_{2}$ we have

$$
\begin{aligned}
& \mathrm{e}^{-\vartheta\left(t-t_{2}\right)} \sum_{m \in \mathbb{Z}} \chi\left(\frac{|m|}{M}\right)\left|\psi_{d m}\left(\tau+t_{2}\right)\right|_{E_{\mu}}^{2} \\
& \leq \mathrm{e}^{-\vartheta\left(t-t_{2}\right)}\left\|\psi_{d}\left(\tau+t_{2}\right)\right\|_{E_{\mu}}^{2} \\
& \leq \mathrm{e}^{-\vartheta\left(t-t_{2}\right)+K_{2} t_{2}}\left\|\psi_{d}(\tau)\right\|_{E_{\mu}}^{2} \\
& \frac{4 \chi_{0}(2 \mu+2 \delta+1)}{M \mu} \int_{\tau+t_{2}}^{\tau+t}\left\|\psi_{d}(s)\right\|_{E_{\mu}}^{2} \mathrm{e}^{-\vartheta(t+\tau-s)} \mathrm{d} s \\
& \leq \frac{4 \chi_{0}(2 \mu+2 \delta+1)}{M \mu}\left\|\psi_{d}(\tau)\right\|_{E_{\mu}}^{2} \\
& \quad \times \int_{\tau+t_{2}}^{\tau} \mathrm{e}^{-\vartheta(t+\tau)+\left(9+K_{2}\right) s-K_{2} \tau} \mathrm{d} s \\
&=\frac{4 \chi_{0}(2 \mu+2 \delta+1)}{M \mu}\left\|\psi_{d}(\tau)\right\|_{E_{\mu}}^{2} \mathrm{e}^{-\vartheta(t+\tau)-K_{2} \tau} \\
& \quad \times \int_{\tau+t_{2}}^{\tau+t} \mathrm{e}^{\left(9+K_{2}\right) s} \mathrm{~d} s \\
& \leq \frac{4 \chi_{0}(2 \mu+2 \delta+1)}{M \mu\left(\vartheta+K_{2}\right)}\left\|\psi_{d}(\tau)\right\|_{E_{\mu}}^{2} \mathrm{e}^{K_{2} t} .
\end{aligned}
$$

Thus, it follows from (62)-(63) that for any $t \geq t_{2}$ and $M>M_{2}\left(\varepsilon, \tau, \mathscr{B}_{0}\right)$,

$$
\begin{aligned}
\sum_{m \in \mathbb{Z}} \chi\left(\frac{|m|}{M}\right)\left|\psi_{d m}(t+\tau)\right|_{E_{\mu}}^{2} & \leq \mathrm{e}^{-\vartheta\left(t-t_{2}\right)+K_{2} t_{2}}\left\|\psi_{d}(\tau)\right\|_{E_{\mu}}^{2} \\
& +\frac{4 \chi_{0}(2 \mu+2 \delta+1)}{M \mu\left(\vartheta+K_{2}\right)}\left\|\psi_{d}(\tau)\right\|_{E_{\mu}}^{2} \mathrm{e}^{K_{2} t} .
\end{aligned}
$$

Pick two sufficient large numbers $T^{*} \geq t_{2}$ and $M^{*}>$ $M_{2}\left(\varepsilon, \tau, \mathscr{B}_{0}\right)$ to satisfy

$$
\mathrm{e}^{-\vartheta\left(T^{*}-t_{2}\right)+K_{2} t_{2}}+\frac{4 \chi_{0}(2 \mu+2 \delta+1)}{M^{*} \mu\left(\vartheta+K_{2}\right)} \mathrm{e}^{K_{2} T^{*}} \doteq \gamma^{2}<\frac{1}{4} .
$$


Then, from (64), we have for $N^{*}>2 M^{*}$ that

$$
\begin{aligned}
& \sum_{|m|>N^{*}}\left|\psi_{d m}\left(T^{*}+\tau\right)\right|_{E_{\mu}}^{2} \\
& \quad \leq \sum_{m \in \mathbb{Z}} \chi\left(\frac{|m|}{M^{*}}\right)\left|\psi_{d m}\left(T^{*}+\tau\right)\right|_{E_{\mu}}^{2} \\
& \leq \gamma^{2}\left\|\psi_{d}(\tau)\right\|_{E_{\mu}}^{2} ;
\end{aligned}
$$

that is,

$$
\begin{aligned}
& \left\|\left(I-P_{N^{*}}\right)\left[U\left(T^{*}+\tau, \tau\right) \psi_{\tau}^{(1)}-U\left(T^{*}+\tau, \tau\right) \psi_{\tau}^{(2)}\right]\right\|_{E_{\mu}} \\
& \quad \leq \gamma\left\|\psi_{\tau}^{(1)}-\psi_{\tau}^{(2)}\right\|_{E_{\mu}}
\end{aligned}
$$

where $\gamma<1 / 2$. The proof is complete.

Now, we can state the main result of this paper.

Theorem 6. Let the conditions of Lemma 4 hold. Then, the process $\{U(t, \tau)\}_{t \geq \tau}$ associated with (21)-(22) possesses a pullback exponential attractor $\{\mathscr{A}(t)\}_{t \in \mathbb{R}}$, satisfying

(1) (compactness and finiteness of dimension) for each $t \in \mathbb{R},\{\mathscr{A}(t)\}$ is a compact set of $E_{\mu}$, and the fractal dimension $\operatorname{dim}_{F} \mathscr{A}(t)$ is finite and uniformly bounded in $t$; that is,

$$
\sup _{t \in \mathbb{R}} \operatorname{dim}_{F} \mathscr{A}(t)<\infty
$$

(2) (positive invariant property) $U(t, \tau) \mathscr{A}(\tau) \subset \mathscr{A}(t)$ for all $t \geq \tau$;

(3) (pullback exponential attractivity) there exist an exponent $\eta>0$ and two positive-valued functions $Q, \mathscr{F}$ : $\mathbb{R}_{+} \mapsto \mathbb{R}_{+}$, such that for any bounded set $\mathscr{B} \subset E_{\mu}$,

$$
\begin{array}{r}
\operatorname{Dist}_{E_{\mu}}(U(t, \tau) \mathscr{B}, \mathscr{A}(t)) \leq Q\left(\|\mathscr{B}\|_{E_{\mu}}\right) \mathrm{e}^{-\eta(t-\tau)}, \\
\tau \in \mathbb{R}, \tau+\mathscr{F}\left(\|\mathscr{B}\|_{E_{\mu}}\right) \leq t<\infty,
\end{array}
$$

where $\operatorname{Dist}_{E_{\mu}}(\cdot, \cdot)$ is the Hausdorff semidistance between two subsets of $E_{\mu}$.

Proof. Using Lemmas 2.3 and 3.1 and Theorem 2 of [30], we obtain the result.

Remark 7. The spectrum of Lyapunov exponents is the most precise tool for identification of the character of motion of a dynamical system [38]. There are some works on the estimation of the dominant Lyapunov exponent of nonsmooth systems by means of synchronization method, one can refer to the articles of Stefański et al. [38-40]. In [38], Stefański and Kapitaniak presented a method to estimate the value of largest Lyapunov exponent both for discrete dynamical systems of known difference equations and also for discrete maps reconstructed from the time evolution of the given system. Following this clue, we can ask naturally the problem that whether the method presented in [38] could be applied to estimate Lyapunov exponents for the trajectories on the pullback attractor $\{\mathscr{A}(t)\}$. It is an interesting and challenging issue for us to investigate.

\section{Acknowledgments}

The authors warmly thank the anonymous referee for useful comments and bringing them the issue on Lyapunov exponents for the trajectories in the pullback attractor into their attention. This paper is supported by National NSFC (no. 11271290), the National Key Basic Research Program of China (973 Program with Grant no. 2012CB426510), and NSF of Wenzhou University (2008YYLQ01).

\section{References}

[1] S. N. Chow, "Lattice dynamical systems," Lecture Notes in Mathematics, vol. 1822, pp. 1-102, 2003.

[2] S.-N. Chow, J. Mallet-Paret, and E. S. Van Vleck, "Pattern formation and spatial chaos in spatially discrete evolution equations," Random \& Computational Dynamics, vol. 4, no. 2-3, pp. 109-178, 1996.

[3] L. O. Chua and T. Roska, "CNN paradigm," IEEE Transactions on Circuits and Systems I, vol. 40, no. 3, pp. 147-156, 1993.

[4] L. O. Chua and L. Yang, "Cellular neural networks: theory," Institute of Electrical and Electronics Engineers. Transactions on Circuits and Systems, vol. 35, no. 10, pp. 1257-1272, 1988.

[5] L. M. Pecora and T. L. Carroll, "Synchronization in chaotic systems," Physical Review Letters, vol. 64, no. 8, pp. 821-824, 1990.

[6] T. Erneux and G. Nicolis, "Propagating waves in discrete bistable reaction-diffusion systems," Physica D, vol. 67, no. 1-3, pp. 237-244, 1993.

[7] R. Kapral, "Discrete models for chemically reacting systems," Journal of Mathematical Chemistry, vol. 6, no. 2, pp. 113-163, 1991.

[8] L. Fabiny, P. Colet, R. Roy, and D. Lenstra, "Coherence and phase dynamics of spatially coupled solid-state lasers," Physical Review A, vol. 47, no. 5, pp. 4287-4296, 1993.

[9] M. Hillert, "A solid-solution model for inhomogeneous systems," Acta Metallurgica, vol. 9, no. 6, pp. 525-535, 1961.

[10] J. P. Keener, "Propagation and its failure in coupled systems of discrete excitable cells," SIAM Journal on Applied Mathematics, vol. 47, no. 3, pp. 556-572, 1987.

[11] P. W. Bates, H. Lisei, and K. Lu, "Attractors for stochastic lattice dynamical systems," Stochastics and Dynamics, vol. 6, no. 1, pp. $1-21,2006$.

[12] Y. Lv and J. Sun, "Dynamical behavior for stochastic lattice systems," Chaos, Solitons and Fractals, vol. 27, no. 4, pp. 10801090, 2006.

[13] P. W. Bates, K. Lu, and B. Wang, "Attractors for lattice dynamical systems," International Journal of Bifurcation and Chaos in Applied Sciences and Engineering, vol. 11, no. 1, pp. 143-153, 2001.

[14] B. Wang, "Dynamics of systems on infinite lattices," Journal of Differential Equations, vol. 221, no. 1, pp. 224-245, 2006.

[15] B. Wang, "Asymptotic behavior of non-autonomous lattice systems," Journal of Mathematical Analysis and Applications, vol. 331, no. 1, pp. 121-136, 2007. 
[16] S. Zhou, "Attractors for second order lattice dynamical systems," Journal of Differential Equations, vol. 179, no. 2, pp. 605-624, 2002.

[17] S. Zhou, "Attractors for first order dissipative lattice dynamical systems," Physica D, vol. 178, no. 1-2, pp. 51-61, 2003.

[18] S. Zhou, "Attractors and approximations for lattice dynamical systems," Journal of Differential Equations, vol. 200, no. 2, pp. 342-368, 2004.

[19] S. Zhou and W. Shi, "Attractors and dimension of dissipative lattice systems," Journal of Differential Equations, vol. 224, no. 1, pp. 172-204, 2006.

[20] A. Y. Abdallah, "Exponential attractors for the first-order lattice dynamical systems," Journal of Mathematical Analysis and Applications, vol. 339, no. 1, pp. 217-224, 2008.

[21] A. Y. Abdallah, "Exponential attractors for second order lattice dynamical systems," Communications on Pure and Applied Analysis, vol. 8, no. 3, pp. 803-813, 2009.

[22] A. Y. Abdallah, "Uniform exponential attractors for first order non-autonomous lattice dynamical systems," Journal of Differential Equations, vol. 251, no. 6, pp. 1489-1504, 2011.

[23] X. Fan, "Exponential attractor for a first-order dissipative lattice dynamical system," Journal of Applied Mathematics, vol. 2008, Article ID 354652, 8 pages, 2008

[24] X. Han, "Exponential attractors for lattice dynamical systems in weighted spaces," Discrete and Continuous Dynamical Systems A, vol. 31, no. 2, pp. 445-467, 2011.

[25] W.-J. Beyn and S. Yu. Pilyugin, "Attractors of reaction diffusion systems on infinite lattices," Journal of Dynamics and Differential Equations, vol. 15, no. 2-3, pp. 485-515, 2003, Special issue dedicated to Victor A. Pliss on the occasion of his 70th birthday.

[26] N. I. Karachalios and A. N. Yannacopoulos, "Global existence and compact attractors for the discrete nonlinear Schrödinger equation," Journal of Differential Equations, vol. 217, no. 1, pp. 88-123, 2005.

[27] E. Van Vleck and B. Wang, "Attractors for lattice FitzHughNagumo systems," Physica D, vol. 212, no. 3-4, pp. 317-336, 2005.

[28] C. Zhao and S. Zhou, "Compact kernel sections for nonautonomous Klein-Gordon-Schrödinger equations on infinite lattices," Journal of Mathematical Analysis and Applications, vol. 332, no. 1, pp. 32-56, 2007.

[29] X. Jia, C. Zhao, and X. Yang, "Global attractor and Kolmogorov entropy of three component reversible Gray-Scott model on infinite lattices," Applied Mathematics and Computation, vol. 218, no. 19, pp. 9781-9789, 2012.

[30] S. Zhou and X. Han, "Pullback exponential attractors for non-autonomous lattice systems," Journal of Dynamics and Differential Equations, vol. 24, no. 3, pp. 601-631, 2012.

[31] I. Fukuda and M. Tsutsumi, "On coupled Klein-GordonSchrödinger equations," The Journal of the Mathematical Society of Japan, vol. 3, pp. 307-321, 1979.

[32] M. Abounouh, O. Goubet, and A. Hakim, "Regularity of the attractor for a coupled Klein-Gordon-Schrödinger system," Differential and Integral Equations, vol. 16, no. 5, pp. 573-581, 2003.

[33] B. Guo and Y. Li, "Attractor for dissipative Klein-GordonSchrödinger equations in $\mathbb{R}^{3}$," Journal of Differential Equations, vol. 136, no. 2, pp. 356-377, 1997.

[34] Y. Li and B. Guo, "Asymptotic smoothing effect of solutions to weakly dissipative Klein-Gordon-Schrödinger equations," Journal of Mathematical Analysis and Applications, vol. 282, no. 1, pp. 256-265, 2003.
[35] K. Lu and B. Wang, "Upper semicontinuity of attractors for the Klein-Gordon-Schrödinger equation," International Journal of Bifurcation and Chaos in Applied Sciences and Engineering, vol. 15, no. 1, pp. 157-168, 2005.

[36] B. Wang and H. Lange, "Attractors for the Klein-GordonSchrödinger equation," Journal of Mathematical Physics, vol. 40, no. 5, pp. 2445-2457, 1999.

[37] S. Zhou and X. Han, "Uniform exponential attractors for nonautonomous KGS and Zakharov lattice systems with quasiperiodic external forces," Nonlinear Analysis. Theory, Methods \& Applications A, vol. 78, pp. 141-155, 2013.

[38] A. Stefański and T. Kapitaniak, "Estimation of the dominant Lyapunov exponent of non-smooth systems on the basis of maps synchronization," Chaos, Solitons and Fractals, vol. 15, no. 2, pp. 233-244, 2003.

[39] A. Stefański, "Estimation of the largest Lyapunov exponent in systems with impacts," Chaos, Solitons \& Fractals, vol. 11, no. 15, pp. 2443-2451, 2000, Dynamics of impact systems.

[40] A. Stefański and T. Kapitaniak, "Using chaos synchronization to estimate the largest Lyapunov exponent of non-smooth systems," Discrete Dynamics in Nature and Society, vol. 4, pp. 207-215, 2000. 


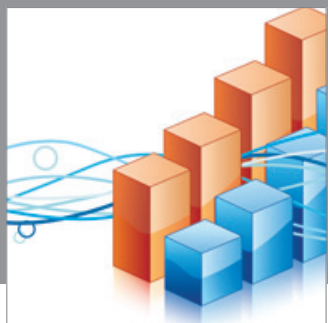

Advances in

Operations Research

mansans

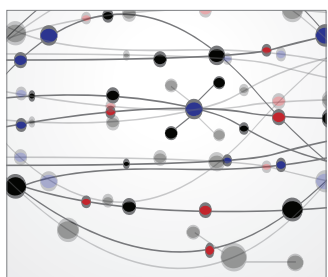

The Scientific World Journal
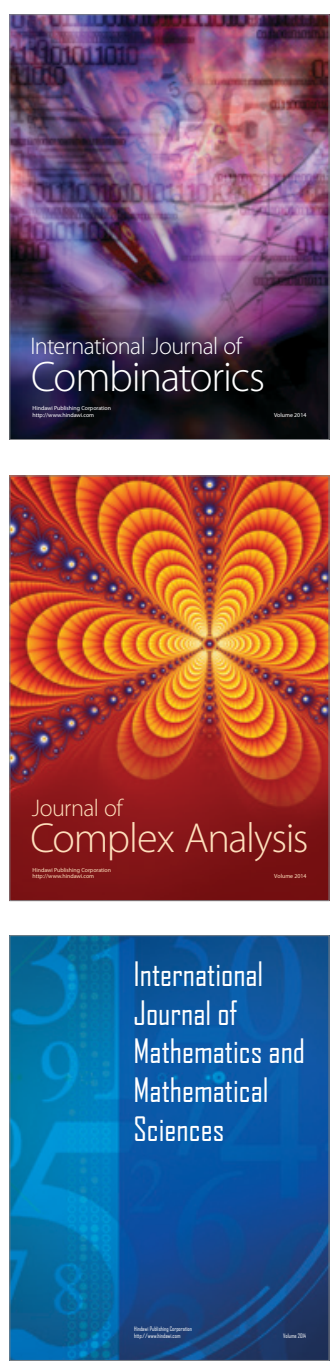
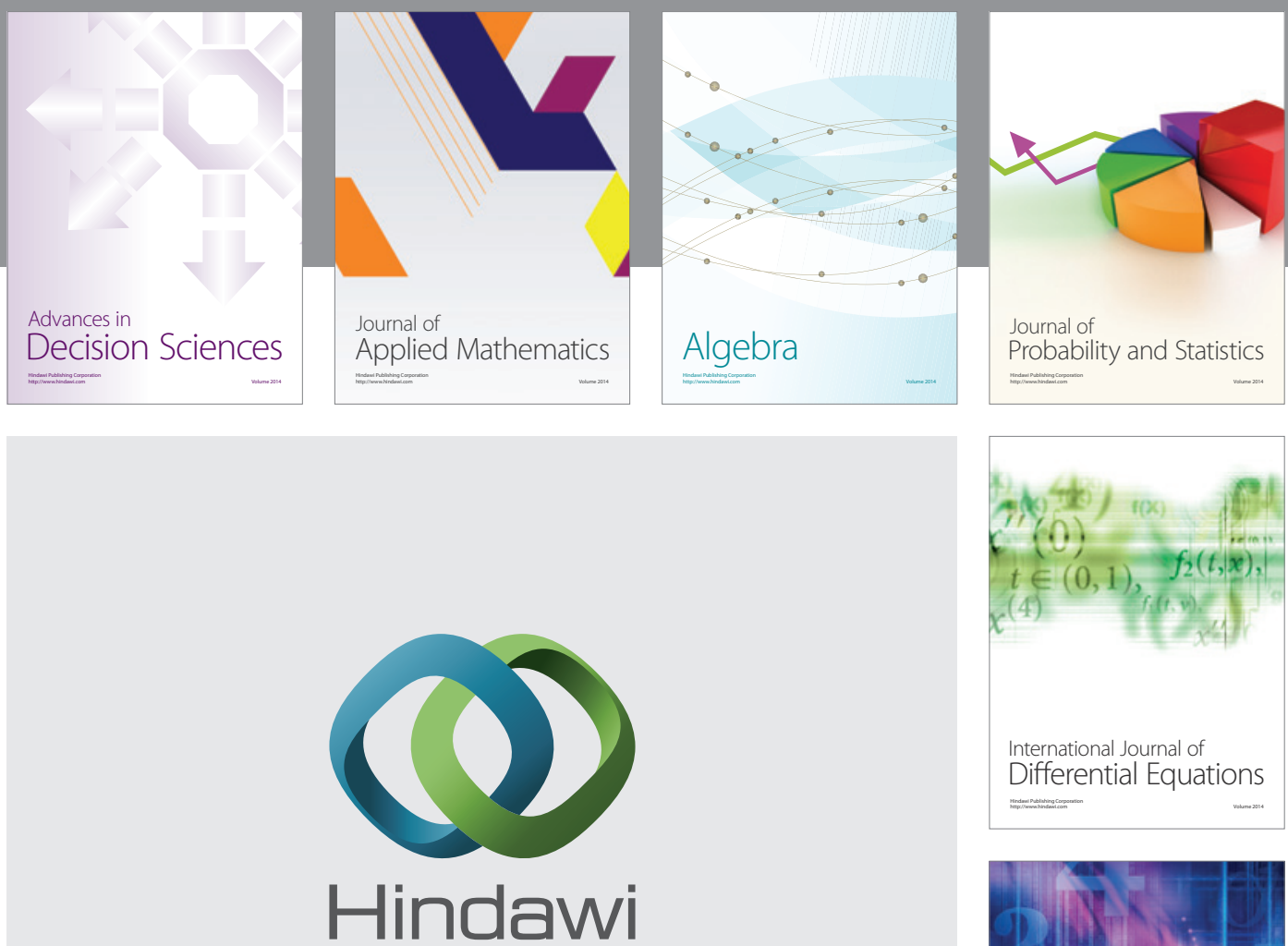

Submit your manuscripts at http://www.hindawi.com
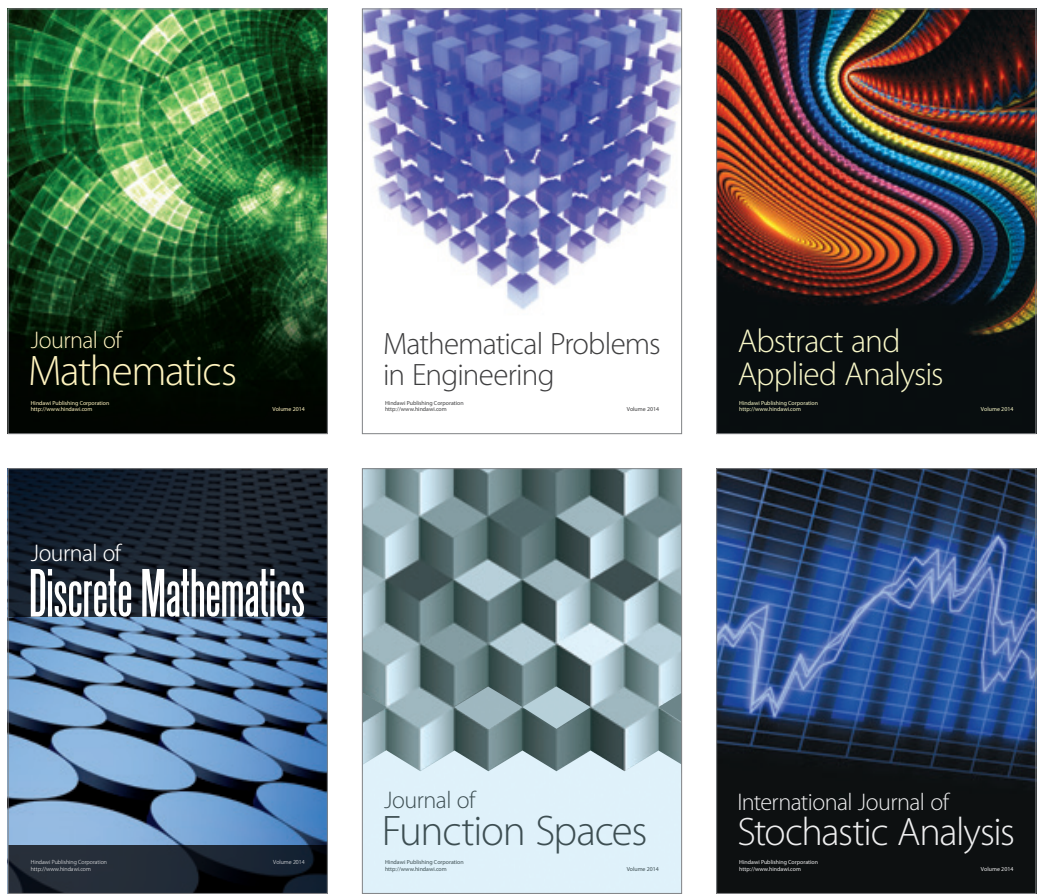

Journal of

Function Spaces

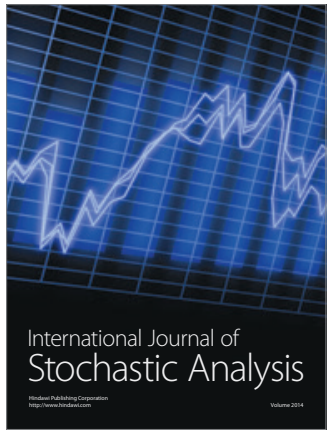

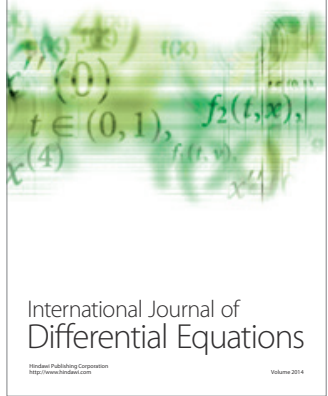
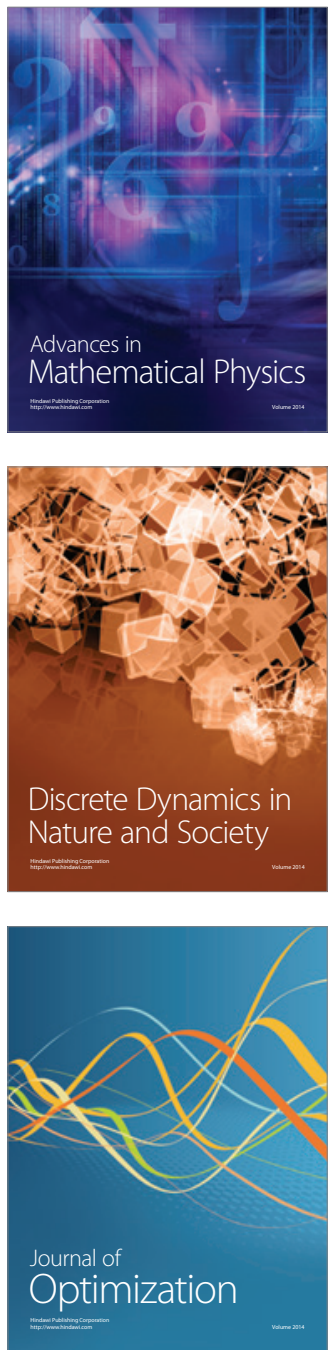